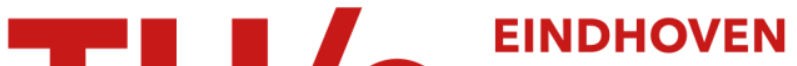 UNIVERSITY OF TECHNOLOGY
}

\section{An experimental study of Faraday waves formed on the interface between two immiscible liquids}

\section{Citation for published version (APA):}

Tipton, C. R., \& Mullin, T. (2004). An experimental study of Faraday waves formed on the interface between two immiscible liquids. Physics of Fluids, 16(7), 2336-2341. https://doi.org/10.1063/1.1718354

DOI:

10.1063/1.1718354

Document status and date:

Published: 01/01/2004

\section{Document Version:}

Publisher's PDF, also known as Version of Record (includes final page, issue and volume numbers)

\section{Please check the document version of this publication:}

- A submitted manuscript is the version of the article upon submission and before peer-review. There can be important differences between the submitted version and the official published version of record. People interested in the research are advised to contact the author for the final version of the publication, or visit the $\mathrm{DOI}$ to the publisher's website.

- The final author version and the galley proof are versions of the publication after peer review.

- The final published version features the final layout of the paper including the volume, issue and page numbers.

Link to publication

\section{General rights}

Copyright and moral rights for the publications made accessible in the public portal are retained by the authors and/or other copyright owners and it is a condition of accessing publications that users recognise and abide by the legal requirements associated with these rights.

- Users may download and print one copy of any publication from the public portal for the purpose of private study or research.

- You may not further distribute the material or use it for any profit-making activity or commercial gain

- You may freely distribute the URL identifying the publication in the public portal.

If the publication is distributed under the terms of Article 25fa of the Dutch Copyright Act, indicated by the "Taverne" license above, please follow below link for the End User Agreement:

www.tue.nl/taverne

Take down policy

If you believe that this document breaches copyright please contact us at:

openaccess@tue.nl

providing details and we will investigate your claim. 


\title{
An experimental study of Faraday waves formed on the interface between two immiscible liquids
}

\author{
C. R. Tipton \\ Physics Department, Eindhoven University of Technology, Postbus 513, 5600 MB Eindhoven, \\ The Netherlands \\ T. Mullin \\ Manchester Centre for Nonlinear Dynamics, University of Manchester, Oxford Road, \\ Manchester M13 9PL, United Kingdom
}

(Received 19 December 2003; accepted 8 March 2004; published online 25 May 2004)

\begin{abstract}
We report results of an experimental study of Faraday waves that were formed on the interface between two immiscible liquids in a cylindrical cell when it was oscillated vertically. The effects of the volume filling ratio $\psi$ on the bifurcation set associated with the onset of the fundamental axisymmetric mode was investigated systematically. In particular, results are presented for the subharmonic regime of the control parameter space where the response was greatest. Both superand subcritical bifurcations are uncovered, with hysteresis in the latter case. The extent of the hysteresis is observed to strongly depend on $\psi$, suggesting that nonlinear damping effects are influenced by this parameter. At large drive amplitudes, a precessional periodic motion was found to develop via a Hopf bifurcation. This mode was observed to disappear catastrophically at an excitation frequency equal to $1.853 \pm 0.006$ times the natural frequency of the resonant mode.

(C) 2004 American Institute of Physics. [DOI: 10.1063/1.1718354]
\end{abstract}

\section{INTRODUCTION}

The onset of waves on the free surface of a liquid that is subjected to a periodic vertical excitation has been the subject of considerable research, and extensive reviews of the literature are provided by Miles and Henderson ${ }^{1}$ and Müller et $a .^{2}$ The phenomenon is often referred to as the Faraday wave instability after Faraday's original observations of waves on the surface of mercury. ${ }^{3}$ More recently, the system has stimulated considerable interest as an example of a pattern-forming system that has rich structure. ${ }^{4}$ Here, we consider a variant of the original free-surface problem by studying the effects on the principal bifurcation structure of adding a second immiscible liquid, as studied theoretically by Kumar and Tuckerman. ${ }^{5}$

In free-surface experiments, at sufficiently small excitation amplitudes, the surface of the liquid is mainly featureless, except for some small ripples associated with the oscillating meniscus. ${ }^{6}$ This essentially flat mode can be related to the trivial state of mathematical models in which the fluid layer is considered to be infinite and the surface is perfectly flat at all drive frequencies. Hence, the liquid layer moves as a solid body in phase with the parametric drive. However, at larger drive amplitudes, this state exchanges stability with one in which waves appear spontaneously on the surface. In experiments, the most commonly observed instability involves a subharmonic mode that arises via a period-doubling bifurcation, although other modes are possible. ${ }^{1,2,7}$ The bifurcation, which gives rise to the subharmonic mode, may be either super- or subcritical, and it is the resulting waves and the bifurcations that give rise to them that form the focus of our study.

An interesting extension to the free-surface problem just described is provided by the vertical excitation of the interface of two immiscible liquid layers, which was first studied theoretically by Kumar and Tuckerman. ${ }^{5}$ They report the results of a Floquet analysis of the linearized Navier-Stokes equations for an interfacial system of infinite lateral extent. The neutral stability curves are calculated and the results compared to a quasi-inviscid model in which a wavenumber-dependent damping term $\gamma$, of the form

$$
\gamma=2 \frac{\left(\mu_{1}+\mu_{2}\right)}{\left(\rho_{1} \operatorname{coth} k h_{1}+\rho_{2} \operatorname{coth} k h_{2}\right)} k^{2}
$$

is added to an extended version of the inviscid theory of Benjamin and Ursell. ${ }^{7}$ (Here, $\mu_{1}, \mu_{2}, \rho_{1}$, and $\rho_{2}$ are the viscosities and densities of the fluids of depths $h_{1}$ and $h_{2}$, respectively, and $k$ is the wave number.) They found that no amount of "tuning" of the fluid parameters enables quantitative agreement between results obtained using the model and those from the full equations of motion. This modified Faraday system provides novel directions for research since the two fluids can be chosen such that they have closely matched densities so that the effective gravitational field can be reduced by up to an order of magnitude. Hence, significantly larger wave amplitudes can be observed compared to the analogous single layer system, and the waves do not break. In our experiment, this reduced gravity ${ }^{8}$ also decreases the required drive frequencies by a third and increases excitation amplitudes by a factor of 60 with respect to the equivalent single-layer setup. This "stretching" of the experimental control parameter space allows a detailed investigation of the bifurcation structure, with experimental control typically within $0.1 \%$.

Our experimental system is small in order to limit the number of available modes. In small-scale experiments, side- 
wall boundaries can play a significant role in determining the dynamics of the surface. The presence of a meniscus leads to parasitic surface ripples being emitted from the sidewalls. ${ }^{6}$ Attempts to minimize or control these effects include pinning, where the surface is pinned at the boundaries, ${ }^{6}$ or using deliberately made soft "beaches" near the rim of the cell. ${ }^{9}$ The practical benefits of these techniques enable cleaner experiments to be performed. However, it is not easy to construct mathematical models of these boundary conditions, so that quantitative comparisons between theory and experiment are difficult. Specifically, extending Kumar and Tuckerman's ${ }^{5}$ work to include the effects of sidewall boundary conditions remains an unsolved and challenging theoretical problem. We therefore chose to use "natural" boundary conditions with the hope that comparisons with available theory can be made.

Previous research on the interfacial arrangement has generally considered the problem as a variant of an internal wave system. ${ }^{10,11}$ All the experimental work to date involves the additional complication of a free surface in addition to the interface between the two fluids. ${ }^{10,12}$ Benielli and Sommeria ${ }^{10}$ report that this free surface remains undisturbed in the parameter regimes they studied. However, the results reported here suggest the form of the upper boundary condition has a significant quantitative effect on the dynamics of the system. In particular, we show that the magnitude of the hysteresis involved in the subcritical bifurcations is strongly dependent on the relative sizes of the fluid layers. As shall be discussed subsequently, the form of this bifurcation structure suggests that the magnitudes of any nonlinear dissipative terms are significantly altered by the proximity of the upper and lower cell walls to the liquid-liquid interface.

We discuss the design and construction of the experimental apparatus and describe the procedures used to obtain the measurements in Sec. II. This is followed by a discussion of the experimental results where we focus on the observed bifurcation structure and compare the results with available theory. We obtain surprisingly good agreement with simple models including collapse of the data obtained with a range of filling fractions. A novel feature of our results is the onset of a precessional mode at a Hopf bifurcation, and this, together with comments on boundary conditions, forms the focus of our discussion.

\section{APPARATUS AND PROCEDURE}

The experimental work reported here was carried out with a fully filled and sealed cylindrical cell. This effectively removed the possibility of post-filling contamination by $\mathrm{mi}-$ croscopic particles so that reproducible results were obtained over periods of months. We concentrate on the measured bifurcation structure associated with the behavior of the fundamental axisymmetric mode, which is shown in the photograph in Fig. 1. The plexiglass cell shown here had dimensions similar to the precision machined stainless steel cell used for the majority of the experiments. The metal cell provided superior boundary conditions over the machined plexiglass cylinder. Moreover, the surface properties of the metal

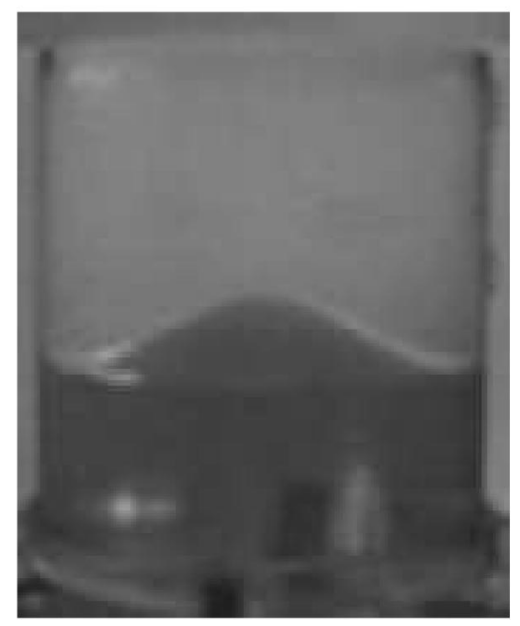

FIG. 1. Side-view photograph of the fundamental axisymmetric mode in a transparent cell of similar dimensions. The water has been colored with ink to aid visualization.

cell remained constant over the entire period of the investigation, whereas it is known that machined plexiglass slowly absorbs water and distorts.

This standing-wave mode had a spatial structure that is well described by inviscid water-wave theory. ${ }^{5,7,8}$ Hence, the interfacial profile is given by $\mathbf{x}(t, r)=\eta(t) J_{0}\left(k_{1} r\right)$, where $\eta(t)$ is some time-dependent amplitude and $J_{0}(k r)$ is a zeroth-order Bessel function of the first kind, with wave number $k_{1} \cdot{ }^{13}$ The radial coordinate $r$ is the distance from the axis of the cell, and a better than $1 \%$ least squares fit of this profile to the data was obtained.

The fluids used were distilled and degassed water and the light silicone oil, hexamethyldisiloxane. The temperature of the environment was maintained at $19.5 \pm 0.4{ }^{\circ} \mathrm{C}$. The water had a density of $997.5 \pm 0.5 \mathrm{~kg} \mathrm{~m}^{-3}$ and a viscosity of $1.033 \pm 0.009 \mathrm{~mm}^{2} \mathrm{~s}^{-1}$, whereas the silicone oil had a density $766.0 \pm 0.5 \mathrm{~kg} \mathrm{~m}^{-3}$ and a viscosity of $0.670 \pm 0.003 \mathrm{~mm}^{2} \mathrm{~s}^{-1}$. These properties were measured using a precision hydrometer and a suspended level viscometer, respectively. The use of these two fluids gave an effective reduced gravity of $(0.1313 \pm 0.007) \mathrm{g}$. The fluids were contained in a machined cylindrical stainless steel cell of diameter $35.22 \pm 0.03 \mathrm{~mm}$. The ends of the cell were formed by two circular glass windows that were sealed onto the steel cylinder by a pair of o-rings.

The lower wall was inserted and a titration pipette was then used to measure the required volume of water into the cell. The remainder of the cell was then completely filled with silicone oil and the upper window clamped in place, ensuring that no air bubbles were trapped within the cell. A filling parameter $\psi$ was calculated such that $\psi=V_{w} / V_{t}$, where $V_{w}$ is the volume of water and $V_{t}$ is the total volume of the cell, which was measured to be $59.0 \pm 0.1 \mathrm{ml}$.

The cell was oscillated sinusoidally and vertically using a scotch-yoke compound bearing driven by a servocontrolled dc electric motor. This allowed the frequency of oscillation to be varied in small steps at a preset fixed amplitude $A$. The excitation was monitored using a linear vari- 


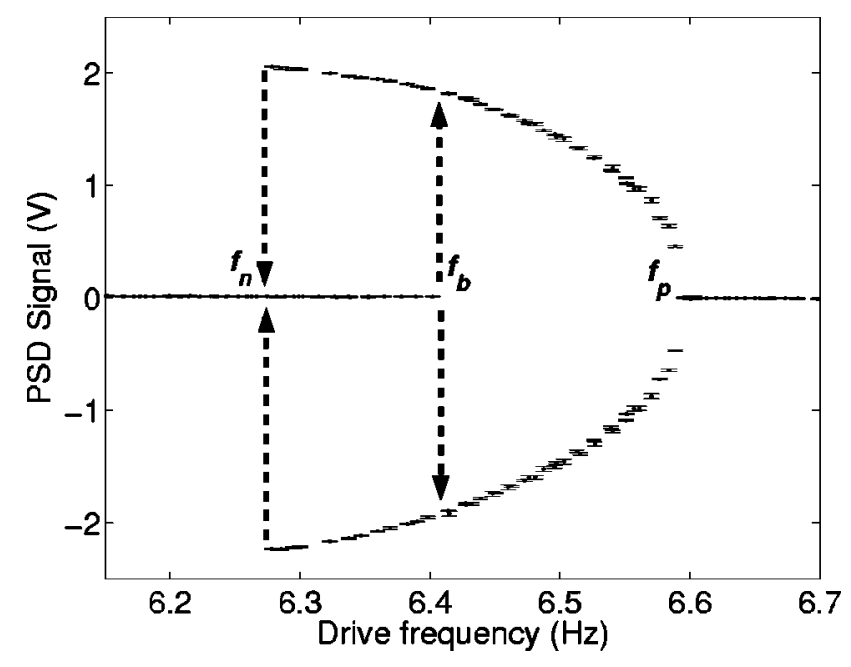

FIG. 2. Bifurcation diagram for period doubling of base state to the fundamental axisymmetric mode. A drive amplitude of $0.990 \pm 0.003 \mathrm{~mm}$ was used, with a filling parameter value of $0.508 \pm 0.006$. Three critical frequencies can be seen: a subcritical period doubling at $f_{b}=6.598 \pm 0.004 \mathrm{~Hz}$, a supercritical period doubling at $f_{p}=6.598 \pm 0.004 \mathrm{~Hz}$, and a saddle node bifurcation at $f_{n}=6.276 \pm 0.004 \mathrm{~Hz}$.

able displacement transformer (LVDT), which produced a voltage signal proportional to the relative height of the platform. Spectral analysis of this displacement signal revealed that the total harmonic content of the drive was less than $0.02 \%$ of the drive.

Surface deformations were monitored using a modified version of a technique first proposed by Gollub and Meyer. ${ }^{14}$ In this study, a laser beam was shone through the cell onto a position-sensitive detector (PSD). The voltage output from the detector was proportional to the gradient of the interface for slopes of up to around $45^{\circ}$. The signal of the LVDT and the PSD were simultaneously sampled using a 12-bit analogue to digital converter and the time series recorded on a computer. Analysis of the dynamical structure was performed using a Poincare section method in which the drive period defined a return time. It was found that return maps constructed from sequences when the drive signal was increasing through zero provided the best signal-to-noise ratio.

The choice of fluids meant that the hydrophobic silicone oil preferentially wet the stainless steel wall and provided a thin oil layer near the junction of the liquid-liquid interface and the cell wall. This allowed the interface to slip and appeared to minimize stick-slip problems at the wall. ${ }^{15}$ Smallamplitude-meniscus driven waves with a frequency equal to the drive were, however, observed below threshold. These waves are caused by the shape of the meniscus changing as a function of time as the effective gravitational field in the cell varied. ${ }^{6}$ The amplitude of the ripples was $\approx 0.1 \mathrm{~mm}$ and were therefore considerably smaller than the subharmonic Faraday waves, which had amplitudes on the order of $10 \mathrm{~mm}$ and did not affect the principal dynamics of the system.

\section{RESULTS}

The fundamental axisymmetric mode shown in Fig. 1 arose at a period doubling bifurcation. The mode was phase

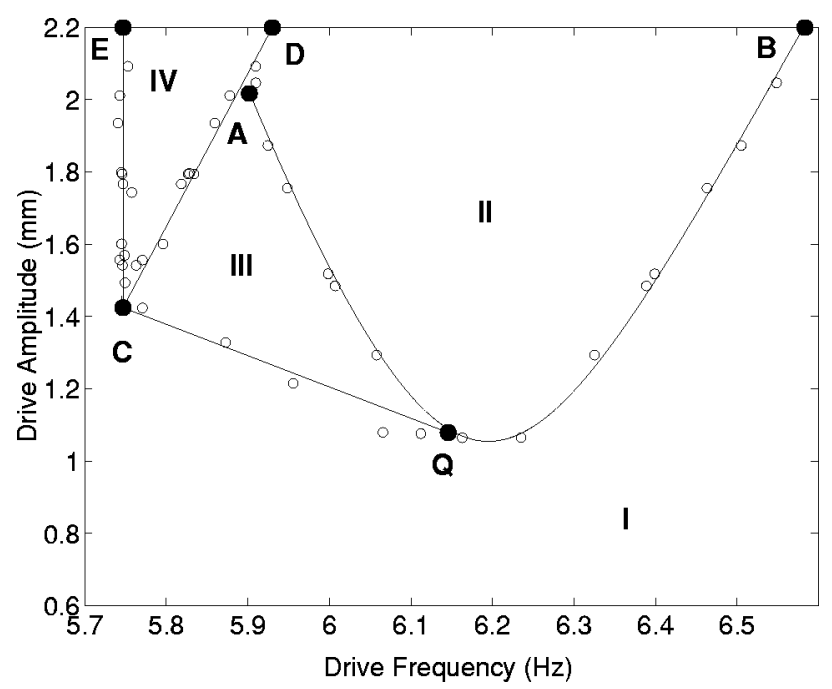

FIG. 3. Experimental stability diagram for a cell with a filling parameter of $\psi=0.878 \pm 0.009$. Region I denotes where the base state is stable; region II, where only the period doubling state is stable; region III, where the period doubling state and the base state are both stable; and region IV, where the precessing state was found. Point $\mathbf{A}$ denotes the position of a codimension-2 point where the Hopf line meets the saddle node line and the periodic fold line. Point $\mathbf{Q}$ designates the position of a quartic point where the primary period doubling bifurcation changes from a supercritical to a subcritical instability.

locked with the drive at precisely half its frequency. An example of a measured typical response diagram is shown in Fig. 2 for a cell with $\psi=0.508 \pm 0.006$ and a drive amplitude of $A=0.990 \pm 0.003 \mathrm{~mm}$. This was constructed by sweeping the drive frequency slowly through a narrow frequency band near $6 \mathrm{~Hz}$ and measuring the response of the PSD as the parametric drive signal increased. The response diagram has two branches since the motion is subharmonic, and hence can be either positive or negative at a chosen point of the drive cycle. The drive frequency was altered in steps of \pm 8.3 $\mathrm{mHz}$ and left to relax for 2 min before sampling the drive and response signals for a further $2 \mathrm{~min}$. These waiting periods were found to be long enough to obtain repeatable estimates of the critical points. The value of the PSD signal at the selected return time was used as the measure of the response, and it is shown plotted as a function of the drive frequency in Fig. 2. A hysteretic region of parameter space was found for drive frequencies less than twice the natural frequency of the fundamental axisymmetric mode. In this example, three distinct critical frequencies can be seen. One is at $f_{b}=6.408 \pm 0.004 \mathrm{~Hz}$, when the base state became unstable via subcritical period doubling bifurcation as the drive frequency is increased. Yet a further increase leads to the restabilization of the base state via a supercritical bifurcation at $f_{p}=6.598 \pm 0.004 \mathrm{~Hz}$. On the other hand, reducing the drive frequency when the axisymmetric mode is present results in the loss of stability of the period doubled state at $f_{n}=6.276 \pm 0.004 \mathrm{~Hz}$. This is a saddle-node bifurcation, and the system returns to the base state after a short transient.

The bifurcation set associated with the onset of the fundamental axisymmetric mode was measured by noting the critical frequencies for the super- and subcritical period doubling bifurcation and saddle-node bifurcation and plotting them as a function of drive frequency for a range of drive 


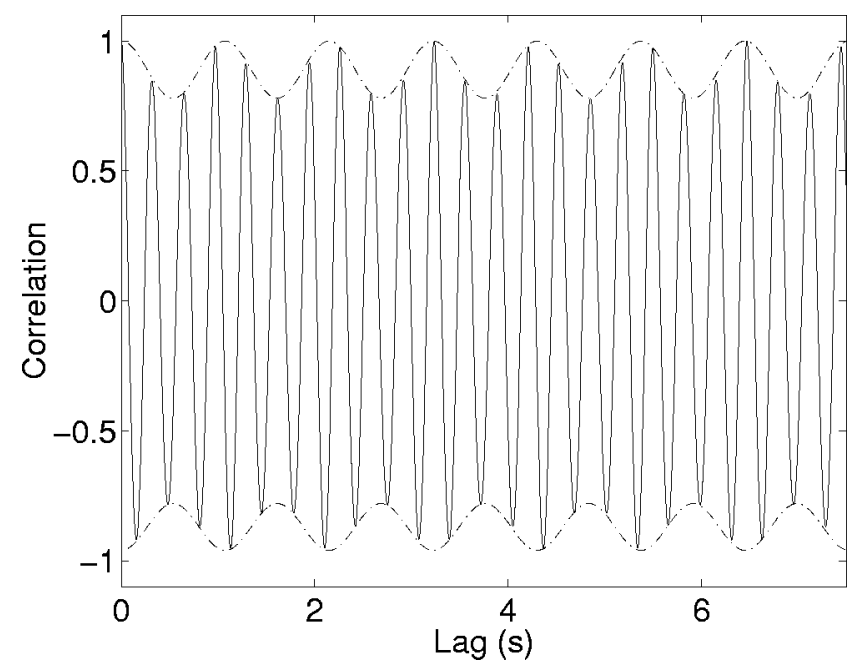

FIG. 4. An example of an experimental autocorrelation function of the PSD signal when the precessing mode was present. In this example the drive frequency was $6.175 \pm 0.003 \mathrm{~Hz}$, the drive amplitude was $1.334 \pm 0.002 \mathrm{~mm}$ and the filling parameter was $0.254 \pm 0.004$. The precessional behavior gives rise to a periodic modulation of the autocorrelation function. The dashed lines correspond to an oscillation with a frequency of $0.93 \mathrm{~Hz}$.

amplitudes and filling parameters. A stability diagram was then constructed by plotting critical points in the frequency amplitude parameter plane. The measured bifurcation set for a cell prepared with a filling parameter of $\psi=0.878 \pm 0.009$ is shown in Fig. 3. Region I denotes the parameter regimes in which only the base state is stable, and region II, enclosed by the curves $\mathbf{A B}$ and $\mathbf{A D}$, denotes where the base state became unstable to the fundamental axisymmetric mode via a period doubling. Region III denotes where the system is bistable, where the period doubled state and the base state coexist and are stable. The point $\mathbf{Q}$ corresponds to a quartic bifurcation point where all three bifurcations met. At this quartic point the period doubling bifurcation changes smoothly from subto supercritical. Hence, the path to the right of $\mathbf{Q}$ is the locus of supercritical period doubling bifurcations, that to the left is subcritical, and $\mathbf{C Q}$ is hence the path of saddle nodes.

Interesting new dynamical behavior was observed in the parameter range labeled IV in Fig. 3. In this region, the period doubled state became unstable via a supercritical Hopf bifurcation to a state in which the lateral position of the maximal interfacial displacement precesses slowly about the center of the cell. The curve $\mathbf{C D}$, which defines the righthand side of region IV, is the locus of these Hopf bifurcations. The frequency of precession was found to be independent of the control parameters and was measured to be 0.93 $\pm 0.05 \mathrm{~Hz}$. An example of an experimental autocorrelation function obtained from the PSD signal when the system was in this precessing state is shown in Fig. 4, and the corresponding experimental Poincaré section is shown in Fig. 5. The vertical line $\mathbf{C E}$ in Fig. 3 defines the left-hand side of region IV and denotes the path of a periodic fold line. Reducing the drive frequency below this critical value causes the precessing state to collapse catastrophically to the base state. The path of periodic folds, Hopf bifurcation points, and SNs meet at point $\mathbf{C}$, which is therefore a codimension-2 point. However, more complicated dynamical motion was

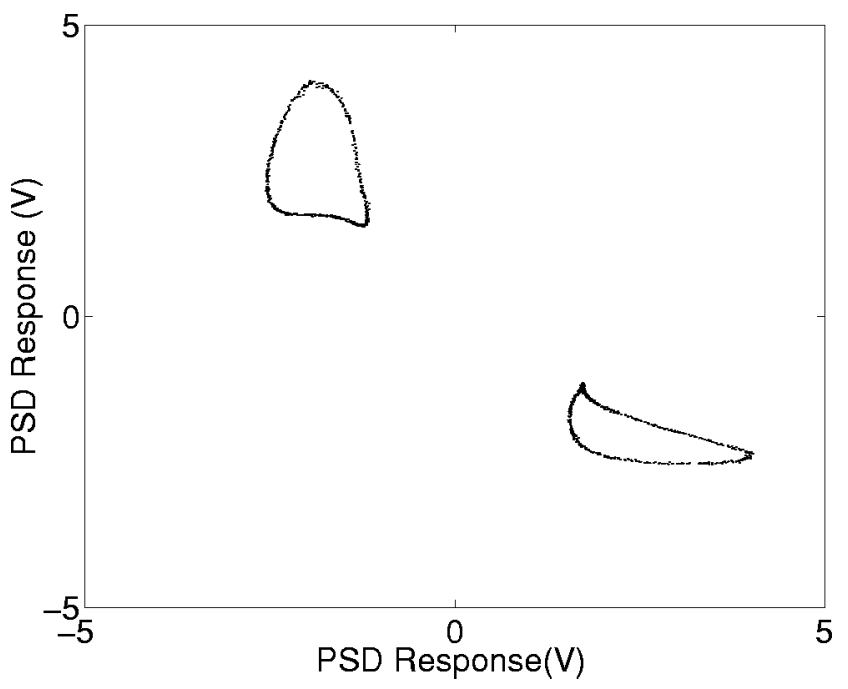

FIG. 5. Experimental Poincaré section obtained from the data used to calculate autocorrelation function shown in Fig. 4. The closed loops signifying the period doubled state has undergone a Hopf bifurcation.

not observed and the period of the oscillation did not show any strong dependence on control parameter in the vicinity of this point.

The stability diagrams were found to be qualitatively the same for all values of $\psi$. However, there were quantitative variations in the critical points with $\psi$. In order to probe them in a systematic manner, the neutral stability curve that defines region II in Fig. 3 was assumed to be given by the marginal stability curve for a subharmonic resonance of the linearly damped Mathieu equation

$$
\ddot{\eta}+\frac{\sqrt{\alpha}}{q} \dot{\eta}+(\alpha+\beta \cos \tau) \eta=0,
$$

where the nondimensional time scale $\tau$ is defined in terms of the drive frequency $f$, as $\tau=2 \pi f t$, the constant $q$ is the linear quality factor of the fundamental axisymmetric mode defined in terms of the natural frequency $f_{0}$ and the linear damping factor $\gamma$, as $q=2 \pi f_{0} / \gamma \cdot{ }^{16}$ The nondimensional control parameters $\alpha$ and $\beta$ are defined as $\alpha=f_{0}^{2} / f^{2}$ and $\beta=A / L$, where $A$ is the experimental drive amplitude and $L$ is a natural length scale related to the wave number of the fundamental axisymmetric mode.

The use of this model does not contradict the findings of Kumar and Tuckerman ${ }^{5}$ since they consider only systems of infinite lateral extent. Here, the small dimensions of the system mean the effect of the sidewalls have a dominating effect on the total dissipation within the cell. Indeed, the estimate of the damping given by Eq. (1) was found to be a factor of 8 too small, for the fluids used here.

The marginal stability curve of Eq. (2) near $\alpha=0.25$ was calculated using the numerical continuation package AUTO 2000. ${ }^{17}$ The experimental values of $f_{0}, q$, and $L$ were then measured using a least squares fit of the linear threshold data to this calculated curve and found to be almost independent of $\psi$.

The variation of the shape and magnitude of the hysteretic region was also investigated as a function of $\psi$. The 


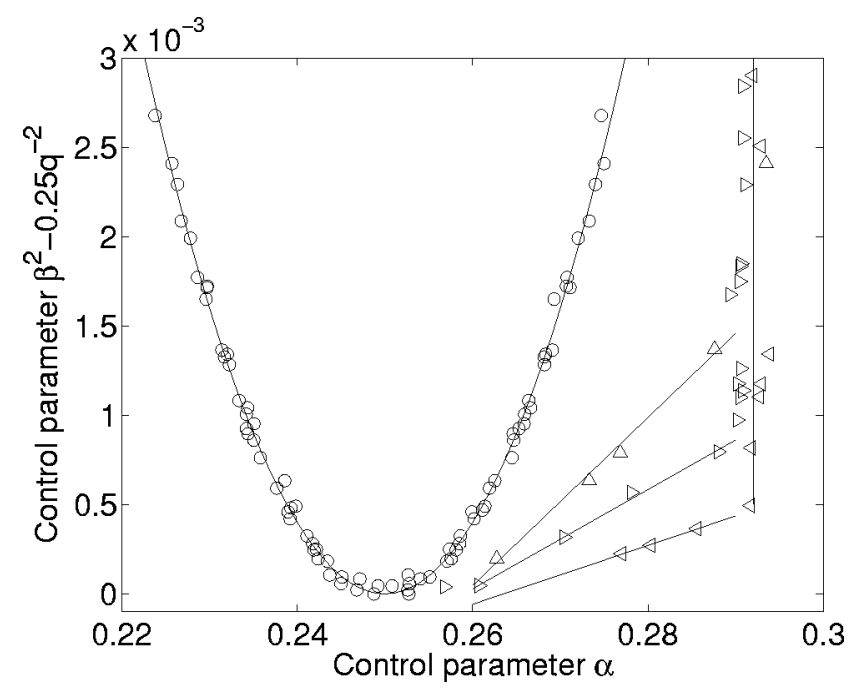

FIG. 6. Stability diagram in terms of the nondimensional control parameters $\alpha$ and $\beta^{2}-0.25 q^{-2}$. The measured neutral stability data for all filling parameters are points marked as $\bigcirc$, these points have been scaled using the fitted parameters $f_{0}, L$, and $q$. The solid curve through these points is the primary subharmonic neutral stability curve of Eq. (2). Also shown is the saddle node and periodic fold data for cells with filling parameters of $\psi=0.085 \pm 0.002$ marked as $\triangle, \psi=0.254 \pm 0.004$ marked as $\triangleleft$, and $\psi=0.848 \pm 0.009$ marked as $\triangleright$. The solid straight lines are shown as a guide to the eye.

experimental stability data were replotted in terms of the nondimensional control parameters $\alpha$ and $\beta^{2}-0.25 q^{-2}$. In these coordinates, the neutral stability data for all filling parameters collapses onto the same linear-order stability curve, allowing the variation of the nonlinear responses to be probed systematically as a function of the nondimensional control parameters. A stability diagram plotted in terms of the nondimensional control parameters is shown in Fig. 6. The data points marked as $\bigcirc$ are the measured neutral stability points for all the experimental data sets. In the chosen coordinate system, these points collapse onto the solid curve, which is the subharmonic neutral stability curve of Eq. (2). The points marked as $\triangle, \triangleleft$, and $\triangleright$ are the saddle node and periodic fold points for data sets with filling parameters equal to $\psi=0.085 \pm 0.002, \psi=0.254 \pm 0.004$, and $\psi=0.848$ \pm 0.009 , respectively.

The periodic fold line corresponding to the parameter values at which the precessing state became unstable and jumped catastrophically to the base state is a vertical line at $\alpha=0.292 \pm 0.002$ for all filling parameters. This corresponds to a drive frequency equal to $f=(1.851 \pm 0.006) f_{0}$. In the nondimensional coordinates, the locus of saddle node points varies linearly with $\alpha$ over the parameter range investigated. Furthermore, the gradient of the saddle node line exhibits a systematic dependence on $\psi$ such that, at filling parameters near the limits $\psi \rightarrow 0$ and $\psi \rightarrow 1$, the gradient becomes very large and there is relatively little hysteresis present. A graph of the observed variation of the saddle-line gradient is shown in Fig. 7 with a solid curve through the data points as a guide to the eye.

The quantitative variation of the saddle node line with $\psi$ is an intrinsically nonlinear phenomena. Numerical investigation of nonlinear variants of Eq. (2) using AUTO 2000 sug-

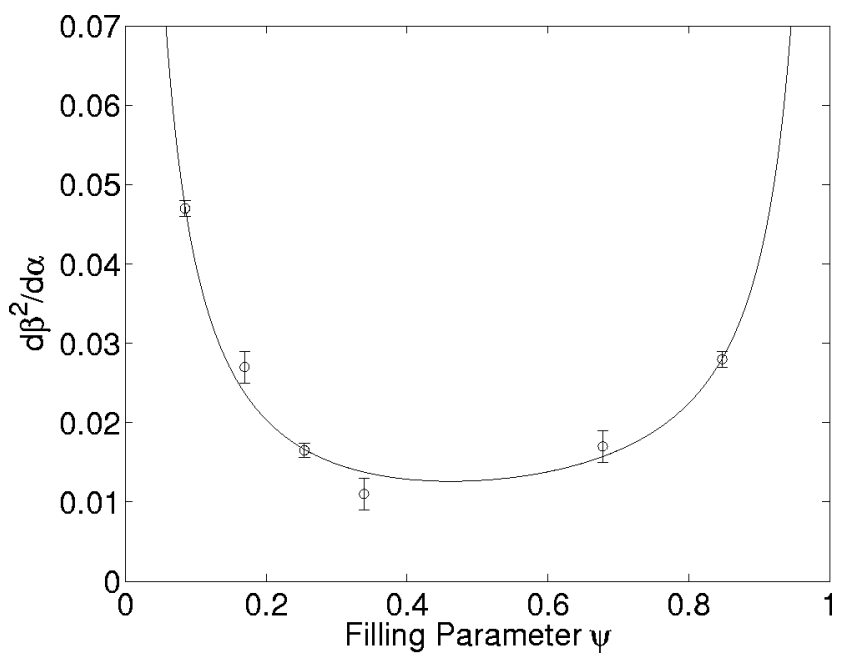

FIG. 7. Variation of saddle node line gradient as a function of filling parameter $\psi$. In the limits $\psi \rightarrow 0$ and $\psi \rightarrow 1$, the gradient of the saddle node line diverges. The solid curve is a guide to the eye.

gested that the variation of the saddle node line gradient is dominated by the form of any dissipative terms in the model equations. This suggests that near the quartic point, the system is essentially governed by a single nonlinear variant of the Mathieu equation with some nonlinear damping function. It is this nonlinear damping function that is chiefly affected by the presence of the end walls of the cell. However, this simple model cannot explain the presence of the precessing state as there are insufficient degrees of freedom in the model for such a state to exist.

\section{CONCLUSIONS}

In summary, we have measured bifurcation curves and stability diagrams associated with the onset of the fundamental axisymmetric mode of an interfacial Faraday waves system. These measurements were used in order to determine linear scaling parameters. At linear order, the measurements were found to be relatively insensitive to the filling parameter of the cell. However, finite amplitude results appeared to indicate that the presence of nonlinear damping within the system was important and varied significantly as a function of filling parameter. A secondary state was found in which the system underwent a Hopf bifurcation and the fundamental axisymmetric mode became unstable to a precessing state. This precessing state became unstable at a critical frequency equal to $f_{c}=(1.851 \pm 0.006) f_{0}$ independent of the filling parameter. This rather surprising result suggests that the position of the periodic fold line is determined primarily by some function of the cell radius, which remained unchanged for all the experimental work done to date. This remains an interesting avenue for further study.

\section{ACKNOWLEDGMENTS}

The work of one of the authors (C.T.) was funded by an EPSRC studentship, and of another (T.M.) by an EPSRC "Senior Fellowship." 
${ }^{1}$ J. Miles and D. Henderson, "Parametrically forced surface waves," Annu. Rev. Fluid Mech. 22, 143 (1990).

${ }^{2}$ H. Müller, R. Friedrich, and D. Papathanassiou, "Theoretical and experimental investigations of the Faraday instability," in Evolution of Spontaneous Structures in Dissipative Continuous Systems, edited by F. Busse and S. Müller (Springer, Berlin, 1998), p. 231.

${ }^{3}$ M. Faraday, "On a peculiar class of accoustical figures; and on certain forms assumed by groups of particles upon vibrating elastic surfaces," Philos. Trans. R. Soc. London 121, 229 (1831).

${ }^{4}$ A. Kudrolli and J. Gollub, "Patterns and spatiotemporal chaos in parametrically forced surface waves: a systematic survey at large aspect ratio," Physica D 97, 133 (1996).

${ }^{5} \mathrm{~K}$. Kumar and L. Tuckerman, "Parametric instability of the interface between two fluids," J. Fluid Mech. 279, 49 (1994).

${ }^{6}$ S. Douady, "Experimental study of the Faraday instability," J. Fluid Mech. 221, 383 (1990).

${ }^{7}$ T. Benjamin and F. Ursell, "The stability of the plane free surface of a liquid in vertical periodic motion," Proc. R. Soc. London, Ser. A 225, 505 (1954).

${ }^{8} \mathrm{H}$. Lamb, Hydrodynamics, 6th ed. (Cambridge University Press, Cambridge, 1932).

${ }^{9}$ A. Wernet, C. Wagner, D. Papathanassiou, H. Müller, and K. Knorr, "Amplitude measurements of Faraday waves," Phys. Rev. E 63, 036305 (2001).
${ }^{10} \mathrm{D}$. Benielli and J. Sommeria, "Excitation and breaking of internal gravity waves by parametric instability," J. Fluid Mech. 374, 117 (1998).

${ }^{11}$ D. Hill, "The Faraday resonance of interfacial waves in weakly viscous fluids," Phys. Fluids 14, 158 (2002).

${ }^{12}$ V. A. Kalinchenko, "Laboratory investigation of parametric instability in a two layer fluid," Izv., Acad. Sci., USSR, Atmos. Oceanic Phys. 22, 155 (1986).

${ }^{13}$ Handbook of Mathematical Functions, edited by M. Abramowitz and I. Stegun (Dover, New York, 1964).

${ }^{14}$ J. Gollub and C. Meyer, "Symmetry-breaking instabilities on a fluid surface," Physica D 6, 337 (1983).

${ }^{15} \mathrm{~T}$. Ito, Y. Tsuki, and Y. Kukita, "Interface waves excited by vertical vibration of stratified fluids in a circular cylinder," J. Nucl. Sci. Technol. 36, 508 (1999).

${ }^{16}$ N. K. Bajaj, The Physics of Waves and Oscillations (Tata McGraw-Hill, New Delhi, 1984).

${ }^{17}$ E. Doedel, R. Paffenroth, A. Champneys, T. Fairgrieve, Y. Kuznetsov, B. Oldeman, B. Sandstede, and X. Wang, "Auto 2000: Continuation and Bifurcation Software for Ordinary Differential Equations (with Homcont)" (Technical Report, California Institute of Technology, Pasadena, CA, 2002) 